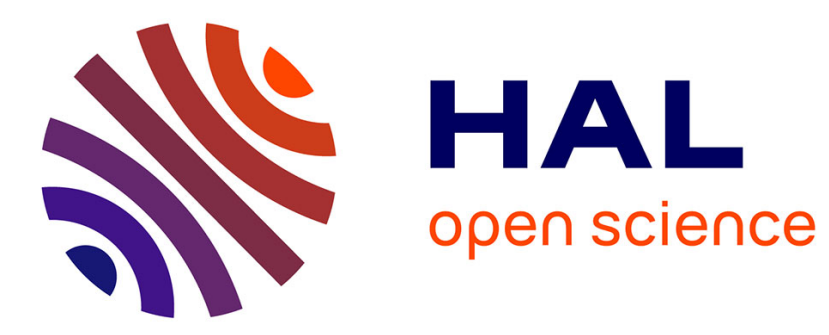

\title{
Cyclic 7-edge-cuts in fullerene graphs
}

František Kardoš, Matjaž Krnc, Borut Lužar, Riste Škrekovski

\section{To cite this version:}

František Kardoš, Matjaž Krnc, Borut Lužar, Riste Škrekovski. Cyclic 7-edge-cuts in fullerene graphs. Journal of Mathematical Chemistry, 2010, 47 (2), pp.771-789. 10.1007/s10910-009-9599-0 . hal00966751

\section{HAL Id: hal-00966751 \\ https://hal.science/hal-00966751}

Submitted on 27 Mar 2014

HAL is a multi-disciplinary open access archive for the deposit and dissemination of scientific research documents, whether they are published or not. The documents may come from teaching and research institutions in France or abroad, or from public or private research centers.
L'archive ouverte pluridisciplinaire HAL, est destinée au dépôt et à la diffusion de documents scientifiques de niveau recherche, publiés ou non, émanant des établissements d'enseignement et de recherche français ou étrangers, des laboratoires publics ou privés. 


\title{
Cyclic 7-edge-cuts in fullerene graphs
}

\author{
František Kardoš ${ }^{a}$, Matjaž Krnc ${ }^{b}$, Borut $\operatorname{Lužar}^{b}$ and Riste Škrekovski ${ }^{b}$
}

${ }^{a}$ Institute of Mathematics, Faculty of Science, P. J. Šafárik University, Jesenná 5, 04001 Košice, Slovakia frantisek.kardos@upjs.sk

${ }^{b}$ Department of Mathematics, University of Ljubljana, Jadranska 19, 1111 Ljubljana, Slovenia

\{matjaz.krnc, borut.luzar\}@gmail.com

\begin{abstract}
A fullerene graph is a planar cubic graph whose all faces are pentagonal and hexagonal. The structure of cyclic edge-cuts of fullerene graphs of sizes up to 6 is known. In the paper we study cyclic 7-edge connectivity of fullerene graphs, distinguishing between degenerated and non-degenerated cyclic edge-cuts, regarding the arrangement of the 12 pentagons. We prove that if there exists a non-degenerated cyclic 7-edge-cut in a fullerene graph, then the graph is a nanotube, unless it is one of the two exceptions presented. We also list the configurations of degenerated cyclic 7-edge-cuts.

keywords: fullerene, fullerene graph, cyclic edge-connectivity, cyclic edge-cut
\end{abstract}

\section{Introduction}

Mathematicians adopted the notion of fullerenes and defined the fullerene graphs as the plane cubic 3-connected graphs with only pentagonal and hexagonal faces. Nanotubes are members of the fullerene structural family. They are cylindrical in shape with the ends typically capped with a hemisphere of the fullerene structure. Nanotubes with the ends left open (open-ended nanotubes) are also interesting objects, see e.g. [8].

Došlić proved that fullerene graphs are cyclically 4-edge connected [2] and cyclically 5-edge connected [3]. The cyclic edge-connectivity of a fullerene graph cannot exceed 5 , since it contains twelve pentagons, thus, there are at least twelve cyclic 5-edge-cuts - formed by the edges pointing outwards of each pentagonal face. There are also cyclic 6 -edge-cuts formed by the edges pointing outwards of each hexagonal face. These cyclic 5- and 6-edge-cuts will be called trivial. Kardoš and Škrekovski [4] characterized 5and 6-edge-cuts, and independently the 5-edge-cuts were characterized by Kutnar and Marušič [6].

An edge-cut of a graph $G$ is a set of edges $C \subset E(G)$ such that $G-C$ is disconnected. A graph $G$ is $k$-edge-connected if $G$ cannot be separated into two components by removing less than $k$ edges. An edge-cut $C$ of a graph $G$ is cyclic if each component of $G-C$ contains a cycle. A graph $G$ is cyclically $k$-edge-connected if $G$ cannot be separated into two components, each containing a cycle, by removing less than $k$ edges. 
A cyclic edge-cut $C$ of a fullerene graph $G$ is non-degenerated, if both components of $G-C$ contain precisely six pentagons. Otherwise, $C$ is degenerated. Obviously, the trivial cyclic edge-cuts are degenerated.

There is a family of fullerene graphs, which have many non-degenerated cyclic edgecuts - the nanotubes. A fullerene graph is a nanotube, if it can be divided into a cylindrical part containing only hexagons, and two caps, each containing six pentagons and some hexagons. The cylindrical part should have the following structure: It contains a ring of hexagons $h_{1}, h_{2}, \ldots, h_{p}$ such that after unfolding it back into the hexagonal grid, there are two unit vectors $a_{1}$ and $a_{2}$ forming a $60^{\circ}$ angle such that each $h_{i}-h_{i-1}$ is either $a_{1}$ or $a_{2}$ for $i=1, \ldots p, h_{0}=h_{p}$. (Here the hexagons are identified with their centers.) In this case, the cylindrical part is an open-ended nanotube of type $\left(p_{1}, p_{2}\right)$, where $p_{j}$ denotes the number of occurences of $a_{j}, j=1,2$. The pair $\left(p_{1}, p_{2}\right)$ of coefficients in the equation $r=p_{1} a_{1}+p_{2} a_{2}$ fully determines the type of the nanotube. It is easy to see that the vectors $a_{1}$ and $a_{2}$ can always be chosen in such a way that $p_{1} \geq p_{2}$.

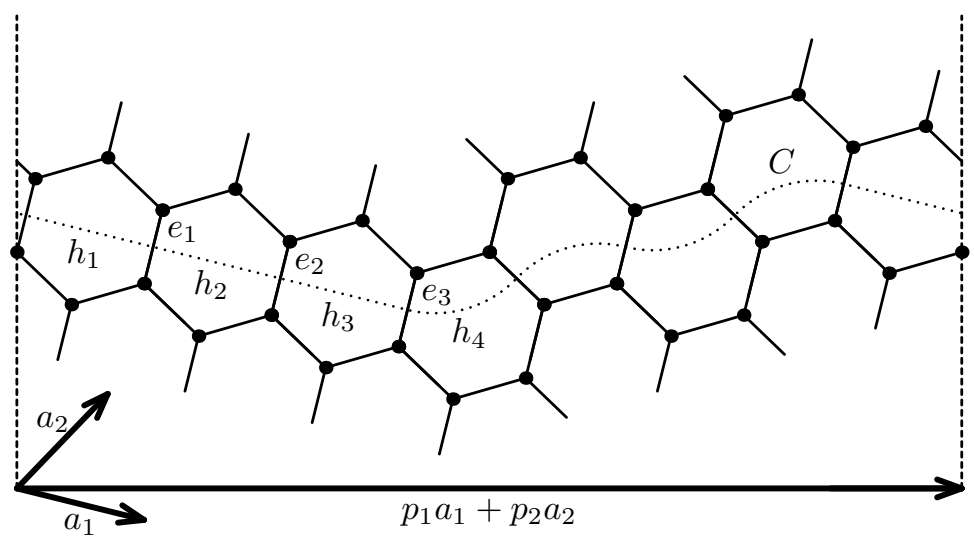

Figure 1: An example of a nanotube of type $(6,2)$.

The nanotubes of types $(n, 0)$ are called zigzag, those of types $(n, n)$ are called armchair (both types have mirror symmetry), the others are chiral (without mirror symmetry). In the light of this definition, also the buckyball $C_{60}$ can be viewed as the first in the series of nanotubes of type $(5,5)$ with a single layer of hexagons in the cylindrical part.

The nanotubes that are interesting in material science usually have the length-todiameter ratio very large. But in many other fullerenes the nanotube-like structure can be found. We say that two non-degenerated cyclic edge-cuts are parallel if both of them induce the two partitions containing the same six pentagons in each, and the corresponding rings of hexagons do not share a face. Such a ring of hexagons is denoted a layer, and the maximal number of parallel layers is the lenght of a nanotube.

It is easy to see that the ring of hexagons induces a non-degenerated cyclic edge-cut in a nanotube. In [4] it was proven that nanotubes are the only graphs having nondegenerated cyclic 5 and 6-edge-cuts, however, there exist graphs that are not nanotubes and have non-degenerated cyclic $k$-edge-cut, for some $k \geq 7$. In the paper we consider non-degenerated cyclic 7-edge-cuts and prove that there exist precisely two fullerenes with non-degenerated cyclic 7-edge-cut, which are not nanotubes. 

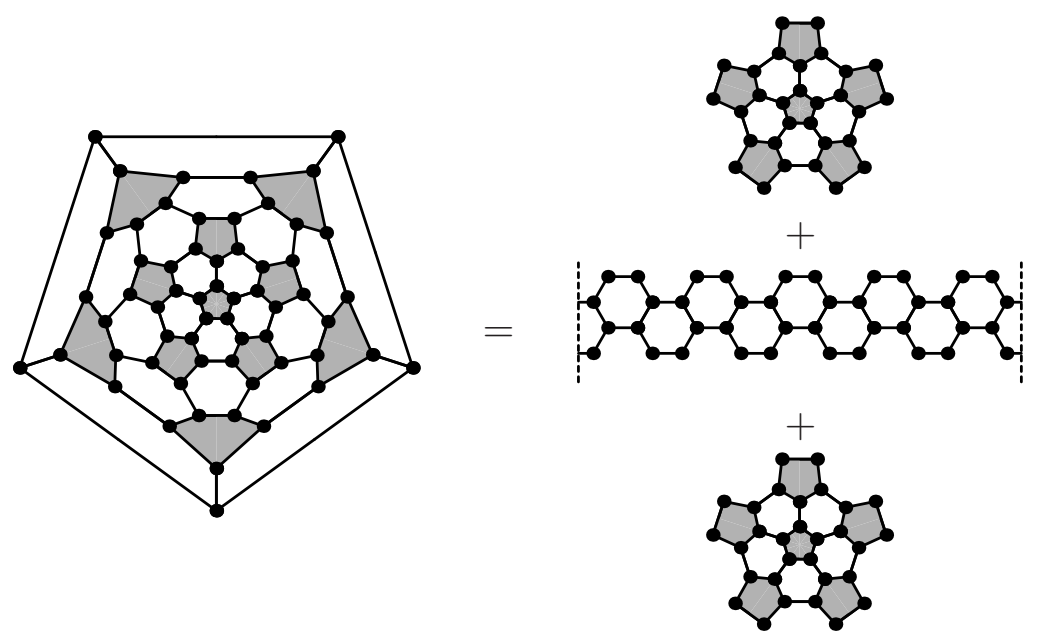

Figure 2: The buckyball is the first nanotube of type $(5,5)$.

Below we pose some known results regarding the non-trivial cyclic 5- and 6-edge-cuts. Let $G_{k}$ denote a fullerene graph comprised of two caps formed by six pentagons, and $k$ layers of hexagons, see Fig. 3.

Theorem 1 A fullerene graph has non-trivial cyclic 5-edge-cut if and only if it is isomorphic to the graph $G_{k}$ for some integer $k \geq 1$.

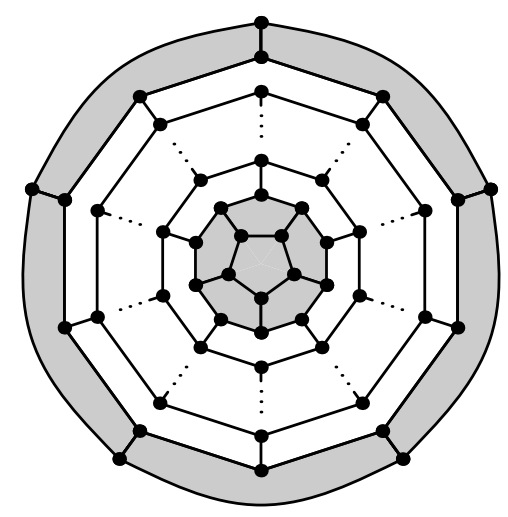

Figure 3: The graphs $G_{k}$ are the only fullerene graphs with non-trivial cyclic 5-edge-cuts.

As an immediate corollary we obtain that all non-trivial cyclic 5-edge-cuts in fullerene graphs are non-degenerated.

An important notion in this paper is a cut-vector. Let $G$ be a fullerene graph and $C$ a $k$-edge cut in $G$, and let $H$ be one of the components of graph $G-C$. We label the vertices of degree one or two in $H$ by $v_{1}, v_{2}, \ldots, v_{k}$. Let $\alpha_{i}$ be the number of 3 -vertices between $v_{i}$ and $v_{i+1}$ (notice that $v_{k+1}=v_{1}$ ) on the segment on the outer face. Note that each vertex $x$ of degree one in $H$ is treated as two 2-vertices $y$ and $w$. We define that between $y$ and $w$ there is -1 vertex of degree three.

We name the sequence $\left[\alpha_{1}, \alpha_{2}, \ldots, \alpha_{k}\right]$ a cut-vector $v(C)$ regarding $H$. It is easy to see that the components $\alpha_{i}$ in fullerenes could only have values $-1,0,1,2$ or 3 , since each face of $G$ is of size 5 or 6 , see Fig. 4 for examples. Observe, that each nondegenerated cyclic edge-cut has two complementary cut-vectors associated with each of 
the components of $G-C$. Here complementary means that knowing one of them is enough, since the sum of components of the same index is in fullerenes always equal to two (only edges of hexagons are elements of non-degenerated cyclic edge-cut). The sum of cut-vector's components has a nice property, which is given in the following lemma:

Lemma 1 Let $C$ be a non-degenerated $k$-cut in a fullerene graph $G$, and let $\alpha=\left[\alpha_{1}, \alpha_{2}\right.$, $\left.\ldots, \alpha_{k}\right]$ be one of its two cut-vectors. Then, $\alpha_{1}+\alpha_{2}+\cdots+\alpha_{k}=k$.

To prove the lemma above, we use an extension of a result from [4, Lemma 1]:

Lemma 2 Let $C$ be an edge-cut in a fullerene graph $G$ and $H$ a component of $G-C$. Let $n_{1}$ and $n_{2}$ be the number of vertices of degree one and two, $f_{5}$ the number of pentagons, and $l$ the size of the outer face of $H$. Then, $6-f_{5}=4 n_{1}+2 n_{2}-l$.

Proof. Let $m$ be the number of edges, $n_{3}$ the number of 3 -vertices, and $f_{6}$ the number of hexagons. Then

$$
n_{1}+2 n_{2}+3 n_{3}=2 m=5 f_{5}+6 f_{6}+l .
$$

Using Euler's formula, we also have that

$$
n_{1}+n_{2}+n_{3}+f_{5}+f_{6}+1-m-2=0 .
$$

Putting it all together we get

$$
\left(2 n_{1}+4 n_{2}+6 n_{3}-4 m\right)+\left(5 f_{5}+6 f_{6}+l-2 m\right)+4 n_{1}+2 n_{2}+f_{5}-l-6=0,
$$

and hence

$$
4 n_{1}+2 n_{2}-l=6-f_{5} .
$$

Proof of Lemma 1. Let $H$ be the component of $G-C$ that corresponds to $\alpha$. By the choice of $C, H$ has $n_{1} 1$-vertices, $n_{2} 2$-vertices, where $2 n_{1}+n_{2}=k$, and six 5 -faces. The length of its outer face is

$$
l=k+\sum_{i=1}^{k} \alpha_{i}=2 n_{1}+n_{2}+\sum_{i=1}^{k} \alpha_{i} .
$$

On the other hand, by Lemma 2 we have

$$
l=4 n_{1}+2 n_{2} .
$$

Hence

$$
\sum_{i=1}^{k} \alpha_{i}=2 n_{1}+n_{2}=k
$$

which proves the lemma. 
The type of a cut-vector $\alpha$ is the vector obtained from $\alpha$ after omitting the components with value 1 . For example, the type of the cut-vector $[2,1,1,0,1,2,0]$ is $[2,0,2,0]$. If no two consecutive components of the cut-vector's type have the same value, we say that the cut is nanotubical. The notion nanotubical derives from the fact, that the two same consecutive components imply that there are all three direction vectors contained in the cut, and we know that the fullerene is a nanotube if and only if there exists a cut containing only two direction vectors.

The notion of cut-vector defined, we can now proceed with cyclic 6-edge-cuts. Unlike cyclic 5-edge-cuts, there exist degenerated cyclic 6-edge-cuts, which are not trivial.

Theorem 2 There are precisely seven non-isomorphic graphs that can be obtained as components of degenerated cyclic 6-edge-cuts with less than six pentagons. Moreover, the graphs with $i$ pentagons are unique for $i=0,1,2,3,4$.

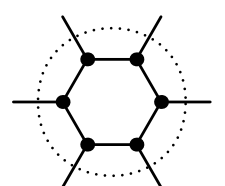

$6 \mathrm{D} 01$

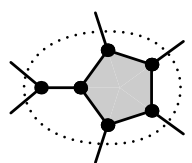

$6 \mathrm{D} 02$

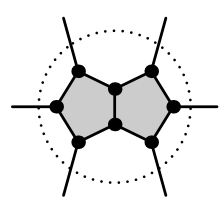

$6 \mathrm{D} 03$

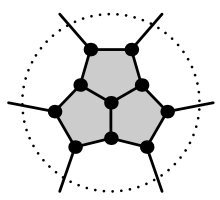

$6 \mathrm{D} 04$

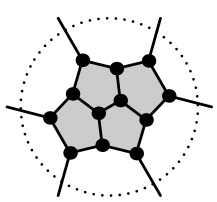

$6 \mathrm{D} 05$

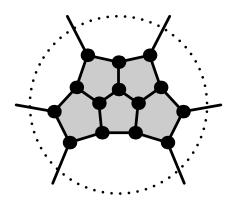

$6 \mathrm{D} 06$

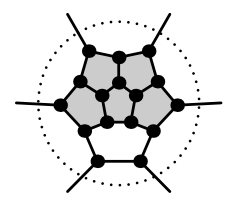

$6 \mathrm{D} 07$

Figure 4: Degenerated cyclic 6-edge-cuts.

Non-degenerated cyclic 6-edge-cuts are, similarly as cyclic 5-edge-cuts, nanotubical. In [4] the following characterization is given:

Theorem 3 A fullerene graph has non-degenerated cyclic 6-edge-cut if and only if it is a nanotube of type $\left(p_{1}, p_{2}\right)$, where

(a) $p_{1}+p_{2}=6 ;$ or

(b) $p_{1}=5, p_{2}=0$, with at least 2 layers of hexagons.

\section{Degenerated cyclic 7-edge-cuts}

In this section we list the degenerated cyclic 7-edge-cuts. There are 57 non-isomorphic graphs that can be obtained as components of degenerate cyclic 7-edge-cuts with less then 6 pentagons. To obtain the configurations we used the reverses of operations $O_{1}$, $\mathrm{O}_{2}$ and $\mathrm{O}_{3}$ presented in [4]. Each of the three operations modifies the cyclic $k$-edge-cut $C$ into another cyclic edge-cut $C_{i}$. Below a brief description of the operations is given (see also Fig.5). 
$\left(O_{1}\right)$ If a component $H$ contains a vertex of degree one, then using $\left(O_{1}\right)$ one can modify the $k$-edge-cut $C$ into a $(k-1)$-edge-cut $C_{1}$.

$\left(\mathrm{O}_{2}\right)$ If a component $H$ contains two adjacent vertices of degree two, then using $\left(\mathrm{O}_{2}\right)$ one can modify the $k$-edge-cut $C$ into a $k$-edge-cut $C_{2}$.

$\left(\mathrm{O}_{3}\right)$ If the vertices of the outer faces of $H$ are consecutively of degree 2 and 3 , then using $\left(\mathrm{O}_{3}\right)$ one can modify the $k$-edge-cut $C$ into a $k$-edge-cut $C_{3}$.
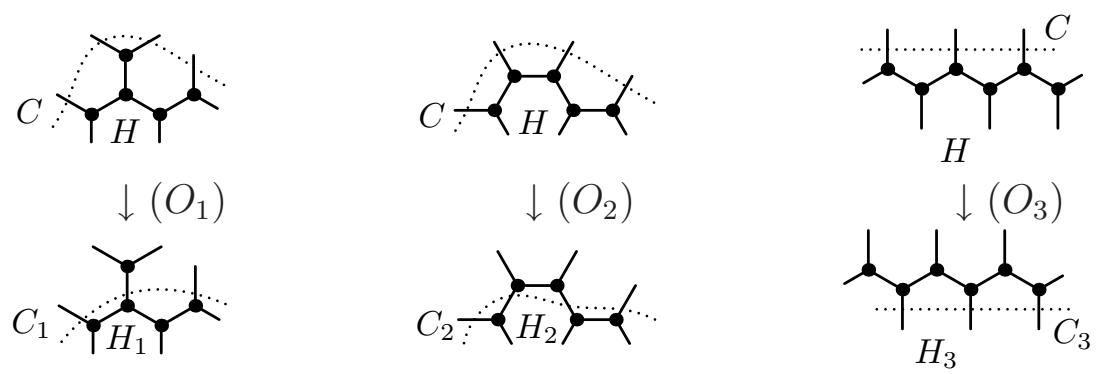

Figure 5: The operations $O_{1}, O_{2}$ and $O_{3}$.

Using the three operations, all cyclic edge-cuts in a fullerene could be constructed, see [4, Theorem 1]. Note that the operation $O_{3}$ can be applied only if there are six pentagons in the configuration $H$, therefore when reconstructing degenerated cyclic edge-cuts from the trivial ones, it is never used. On Fig. 6 an example of constructing a degenerate cyclic 7-edge-cut is presented, and on Fig. 7 we listed the degenerated cyclic 7-edge-cuts.

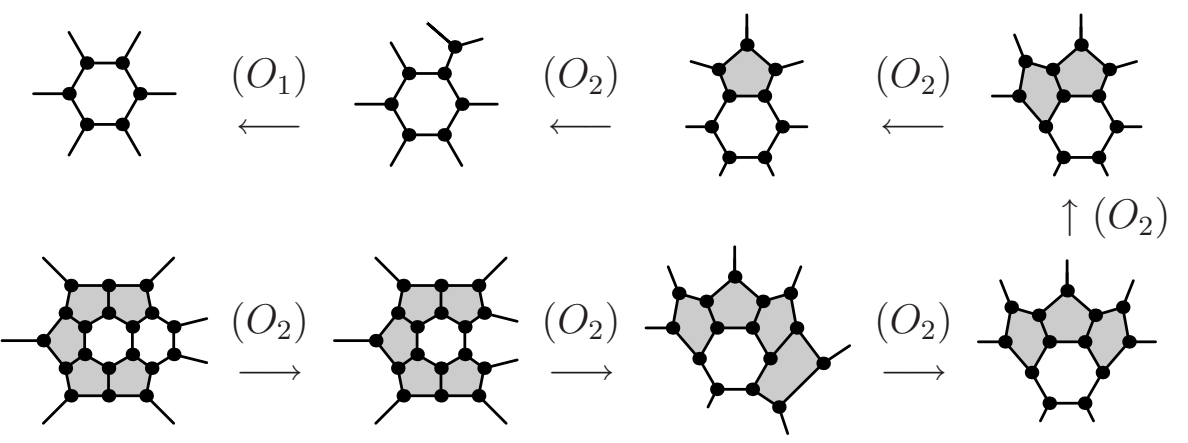

Figure 6: An example of construction.

On Table 1 for each configuration depicted on Fig. 7 we list the number of pentagonal and hexagonal faces (denoted by $f_{5}$ and $f_{6}$ ), the number of vertices (denoted by $v$ ), the cut-vector, and the configurations that arise when applying operations $O_{1}, O_{2}$ and an inverse $\mathrm{O}_{2}^{-1}$.

\section{Non-degenerated cyclic 7-edge-cuts}

In this section, we consider the non-degenerated cyclic 7-edge-cuts. We prove that all non-degenerated cyclic 7-edge-cuts are contained in fullerene graphs which are nanotubes, with two exceptions. There exist precisely two fullerene graphs, which have non-degenerated cyclic 7 -edge-cuts and that are not nanotubical. We also characterize 


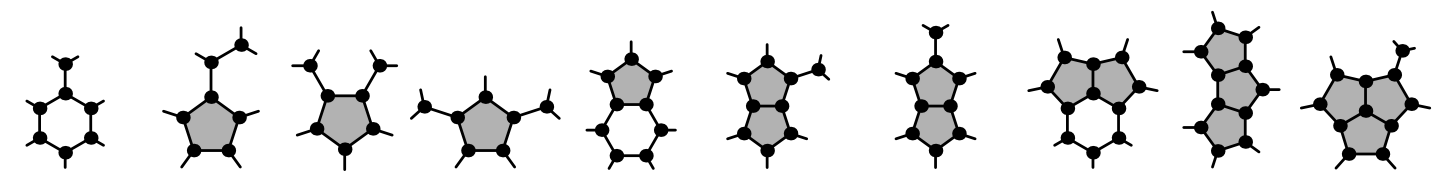
D01 D02 D03 D04 D05 D06 D07 D08 D09 D10

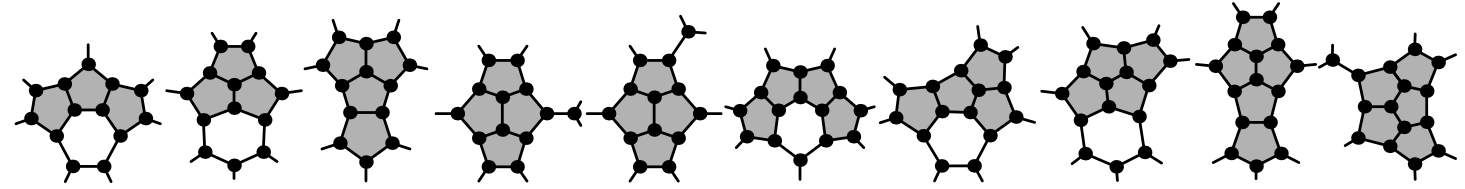
D11
D12 D13
D14 D15
D16
D17
D18
D19 D20
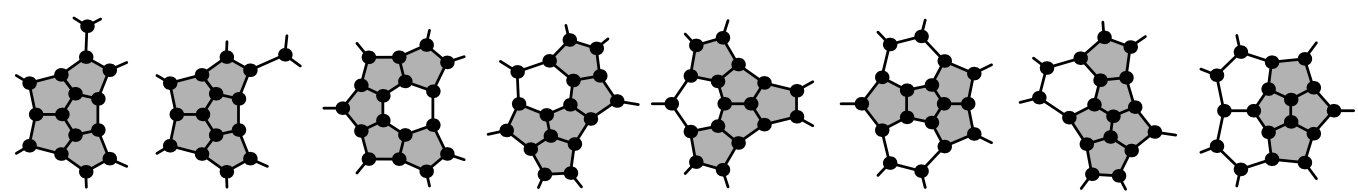

D2

D22

D23

D24

D25

D26

D27

D28
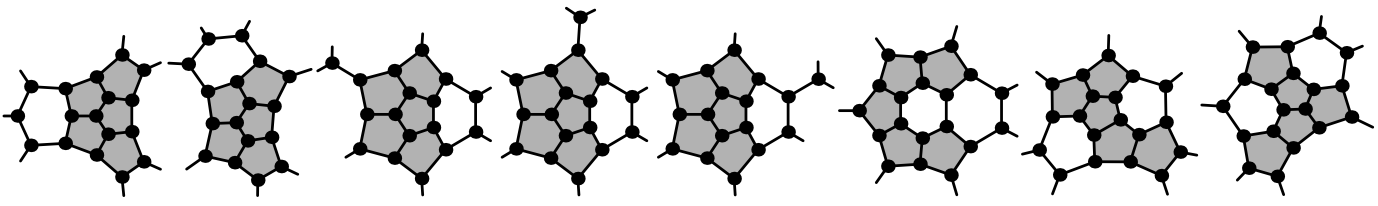

D29

D30

D31

D32

D33

D34

D35

D36

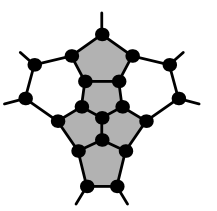

D37

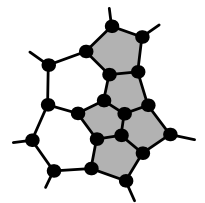

D38

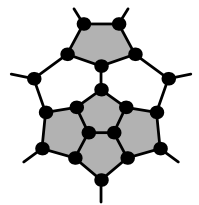

D39

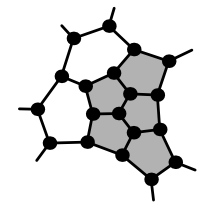

D40

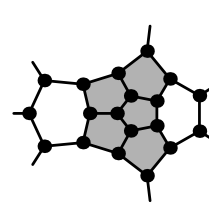

D41

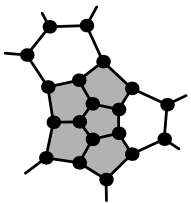

D42

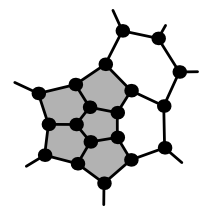

D43

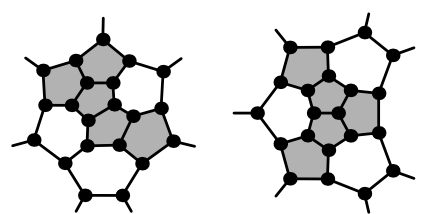

D44

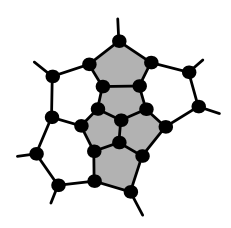

D46

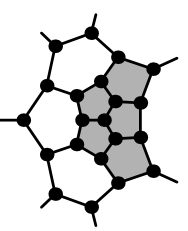

D47

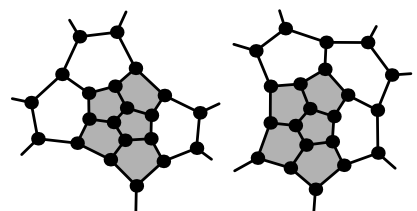

D49

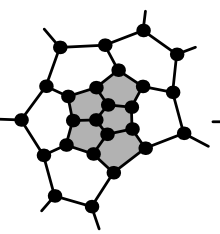

D55

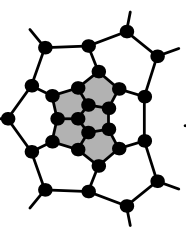

D56

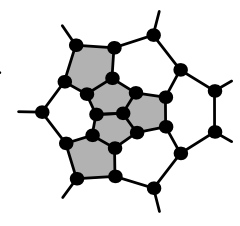

D50

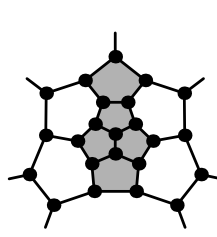

D51

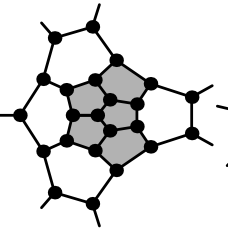

D52

D53

D54

Figure 7: Degenerated cyclic 7-edge-cuts. 


\begin{tabular}{|c|c|c|c|c|c|c|c|}
\hline cut & $f_{5}$ & $f_{6}$ & $v$ & cut-vector & $O_{1}$ & $\mathrm{O}_{2}$ & $\mathrm{O}_{2}^{-1}$ \\
\hline$\overline{\text { D01 }}$ & 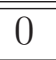 & 1 & 7 & {$[-1,1,0,0,0,0,1]$} & 6D01 & $\begin{array}{c}- \\
\end{array}$ & D05 \\
\hline D02 & 1 & 0 & 7 & {$[-1,0,1,0,0,0,2]$} & $6 \mathrm{D} 02$ & - & D05, D06 \\
\hline D03 & 1 & 0 & 7 & {$[-1,1,0,0,1,-1,2]$} & $6 \mathrm{D} 02$ & - & D05, D06 \\
\hline D04 & 1 & 0 & 7 & {$[-1,1,0,1,-1,1,1]$} & $6 \mathrm{D} 02$ & - & D06, D07 \\
\hline$\overline{\mathrm{D} 05}$ & 1 & 1 & 9 & $\overline{[0,0,0,1,0,0,1]}$ & $\begin{array}{c}- \\
\end{array}$ & D02, D03 & D08 \\
\hline D06 & 2 & 0 & 9 & {$[-1,1,0,1,0,0,2]$} & $6 \mathrm{D} 03$ & D02, D03, D04 & D08, D09, D10 \\
\hline D07 & 2 & 0 & 9 & {$[-1,1,1,0,0,1,1]$} & $6 \mathrm{D} 03$ & D03 & D09, D10 \\
\hline D08 & 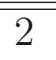 & 1 & 11 & $\overline{[0,0,1,0,1,0,1]}$ & - & D05, D06 & D11, D12 \\
\hline D09 & 3 & 0 & 11 & {$[0,0,1,1,0,0,2]$} & - & D06, D07 & D11, D13 \\
\hline D10 & 3 & 0 & 11 & {$[-1,1,1,0,1,0,2]$} & $6 \mathrm{D} 04$ & D06, D07 & $\mathrm{D} 12, \mathrm{D} 13, \mathrm{D} 14, \mathrm{D} 15$ \\
\hline D11 & 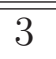 & $\overline{11}$ & 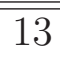 & 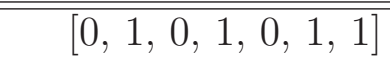 & 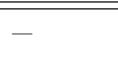 & 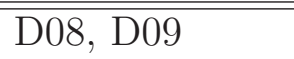 & 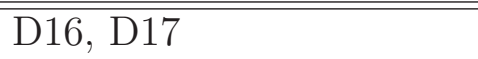 \\
\hline D12 & 3 & 1 & 13 & {$[0,0,1,1,0,1,1]$} & - & D08, D10 & D17, D18 \\
\hline D13 & 4 & 0 & 13 & {$[0,0,2,0,1,0,2]$} & 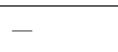 & D09, D10 & D17, D19 \\
\hline D14 & 4 & 0 & 13 & {$[-1,2,0,1,1,0,2]$} & $6 \mathrm{D} 05$ & D09 & D18, D20 \\
\hline D15 & 4 & 0 & 13 & {$[-1,1,1,1,0,1,2]$} & $6 \mathrm{D} 05$ & D09 & D18, D19, D20, D21, D22 \\
\hline D16 & 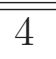 & 1 & 15 & $\overline{[0,1,1,0,1,1,1]}$ & - & D11 & D23, D24, D25 \\
\hline D17 & 4 & 1 & 15 & {$[0,1,0,1,1,0,2]$} & - & $\mathrm{D} 11, \mathrm{D} 12, \mathrm{D} 13$ & D24, D25, D26, D27 \\
\hline D18 & 4 & 1 & 15 & {$[0,0,1,1,1,0,2]$} & - & $\mathrm{D} 12, \mathrm{D} 14, \mathrm{D} 15$ & D27, D28, D29, D30 \\
\hline D19 & 5 & 0 & 15 & {$[0,0,2,1,0,1,2]$} & - & $\mathrm{D} 13, \mathrm{D} 15$ & D27 \\
\hline D20 & 5 & 0 & 15 & {$[-1,2,0,2,0,1,2]$} & $6 \mathrm{D} 06$ & $\mathrm{D} 14, \mathrm{D} 15$ & D29, D30, D31 \\
\hline D21 & 5 & 0 & 15 & {$[-1,1,2,0,1,1,2]$} & $6 \mathrm{D} 06$ & D15 & D30, D32 \\
\hline $\mathrm{D} 22$ & 5 & 0 & 15 & {$[-1,1,1,1,1,0,3]$} & $6 \mathrm{D} 06$ & D15 & - \\
\hline 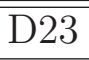 & 5 & 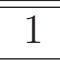 & 17 & $\overline{[0,1,1,1,1,0,2]}$ & 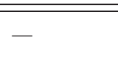 & 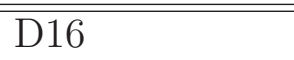 & D34 \\
\hline D24 & 5 & 1 & 17 & {$[0,1,1,1,0,1,2]$} & - & D16, D17 & D35 \\
\hline D25 & 5 & 1 & 17 & {$[0,1,1,0,2,0,2]$} & - & D16, D17 & D36 \\
\hline D26 & 4 & 2 & 17 & {$[0,1,1,0,1,1,1]$} & - & D17 & D35, D36, D37 \\
\hline D27 & 5 & 1 & 17 & {$[0,1,0,2,0,1,2]$} & - & D17, D18, D19 & D37, D38 \\
\hline D28 & 4 & 2 & 17 & {$[0,1,0,1,1,1,1]$} & - & D18 & D38, D39, D40 \\
\hline D29 & 5 & 1 & 17 & {$[0,0,2,0,2,0,2]$} & - & $\mathrm{D} 18, \mathrm{D} 20$ & $\mathrm{D} 40, \mathrm{D} 41$ \\
\hline D30 & 5 & 1 & 17 & {$[0,0,1,2,0,1,2]$} & - & $\mathrm{D} 18, \mathrm{D} 20, \mathrm{D} 21$ & $\mathrm{D} 40, \mathrm{D} 42$ \\
\hline D31 & 5 & 1 & 17 & {$[-1,2,1,0,1,1,2]$} & $6 \mathrm{D} 07$ & $\mathrm{D} 20$ & $\mathrm{D} 41, \mathrm{D} 42$ \\
\hline D32 & 5 & 1 & 17 & {$[-1,2,0,1,1,1,2]$} & $6 \mathrm{D} 07$ & D21 & $\mathrm{D} 42, \mathrm{D} 43$ \\
\hline D33 & 5 & 1 & 17 & {$[-1,1,1,1,1,1,2]$} & $6 \mathrm{D} 07$ & - & D43 \\
\hline D34 & 5 & 2 & 19 & {$[0,1,1,1,1,1,1]$} & - & D23 & - \\
\hline D35 & 5 & 2 & 19 & {$[0,1,1,1,1,0,2]$} & - & D24, D26 & D44 \\
\hline D36 & 5 & 2 & 19 & {$[0,1,1,1,0,1,2]$} & - & D26, D25 & $\mathrm{D} 45$ \\
\hline D37 & 5 & 2 & 19 & {$[0,1,1,0,2,0,2]$} & - & D26, D27 & D46 \\
\hline D38 & 5 & 2 & 19 & {$[0,1,1,0,1,1,2]$} & - & D27, D28 & D46 \\
\hline D39 & 5 & 2 & 19 & {$[0,1,1,1,1,1,1]$} & - & D28 & - \\
\hline D40 & 5 & 2 & 19 & {$[0,1,0,1,2,0,2]$} & - & D28, D29, D30 & $\mathrm{D} 47, \mathrm{D} 48$ \\
\hline D41 & 5 & 2 & 19 & {$[0,0,2,1,0,1,2]$} & - & D29, D31 & D48 \\
\hline $\mathrm{D} 42$ & 5 & 2 & 19 & {$[0,0,2,0,1,1,2]$} & - & D30, D31, D32 & D48, D49 \\
\hline D43 & 5 & 2 & 19 & {$[0,0,1,1,1,1,2]$} & - & D32, D33 & D49 \\
\hline
\end{tabular}




\begin{tabular}{|l||l|l|l|l|l|l|l|}
\hline cut & $f_{5}$ & $f_{6}$ & $v$ & vector & $O_{1}$ & $O_{2}$ & $O_{2}^{-1}$ \\
\hline \hline D44 & 5 & 3 & 21 & {$[0,1,1,1,1,1,1]$} & - & D35 & - \\
\hline D45 & 5 & 3 & 21 & {$[0,1,1,1,1,0,2]$} & - & D36 & D50 \\
\hline D46 & 5 & 3 & 21 & {$[0,1,1,1,0,1,2]$} & - & D37, D38 & D51 \\
\hline D47 & 5 & 3 & 21 & {$[0,1,1,0,1,2,1]$} & - & D40 & D52 \\
\hline D48 & 5 & 3 & 21 & {$[0,1,0,2,0,1,2]$} & - & D40, D41, D42 & D52, D53 \\
\hline D49 & 5 & 3 & 21 & {$[0,1,0,1,1,1,2]$} & - & D42, D43 & D53 \\
\hline \hline D50 & 5 & 4 & 23 & {$[0,1,1,1,1,1,1]$} & - & D45 & - \\
\hline D51 & 5 & 4 & 23 & {$[0,1,1,1,1,0,2]$} & - & D46 & D54 \\
\hline D52 & 5 & 4 & 23 & {$[0,1,1,0,2,0,2]$} & - & D47, D48 & D55 \\
\hline D53 & 5 & 4 & 23 & {$[0,1,1,0,1,1,2]$} & - & D48, D49 & D55 \\
\hline \hline D54 & 5 & 5 & 25 & {$[0,1,1,1,1,1,1]$} & - & D51 & - \\
\hline D55 & 5 & 5 & 25 & {$[0,1,1,1,0,1,2]$} & - & D52, D53 & D56 \\
\hline \hline D56 & 5 & 6 & 27 & {$[0,1,1,1,1,0,2]$} & - & D55 & D57 \\
\hline \hline D57 & 5 & 7 & 29 & {$[0,1,1,1,1,1,1]$} & - & D56 & - \\
\hline
\end{tabular}

Table 1: Degenerated cyclic 7-edge cuts.

the types of nanotubes in which non-degenerate cyclic 7-edge-cuts exist. It is obvious that nanotubes of type $\left(p_{1}, p_{2}\right)$, where $p_{1}+p_{2} \geq 8$, cannot contain such a cut, due to the width of the cylindrical part (of course, degenerate cyclic edge-cuts are not limited by the type).

Regarding nanotube types, where the sum $p_{1}+p_{2}<7$, it was already proven in [4] that only graphs $G_{k}$ contain non-trivial cyclic 5-edge-cuts, in other words, for $p_{1}+p_{2}=5$, only nanotubes of type $(5,0)$ exist. On the other hand, there are more possible types for $p_{1}+p_{2}=6$. For type $(6,0)$ there exist five different caps, while for types $(5,1),(4,2)$, and $(3,3)$ caps are unique. On Fig. 8 the caps retrieved from nanotubes of specified types are presented. Note that nanotubes with $p_{1}+p_{2}<5$ do not exist, due to cyclic 5-edge-connectivity of fullerenes.

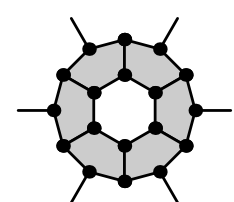

$(6,0)$

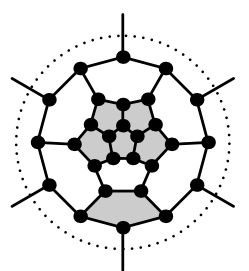

$(6,0)$

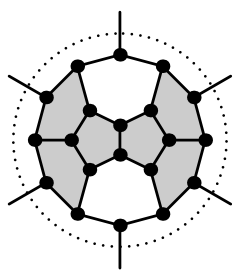

$(6,0)$

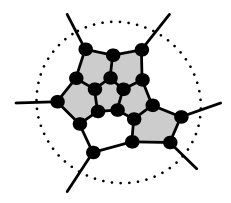

$(5,1)$

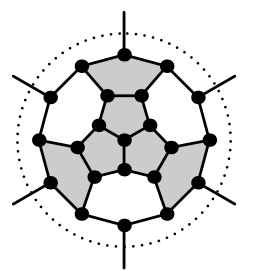

$(6,0)$

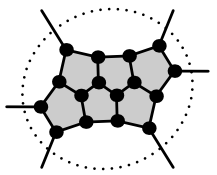

$(4,2)$

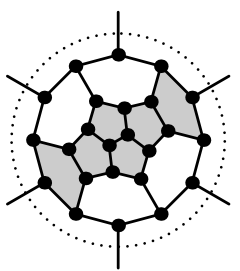

$(6,0)$

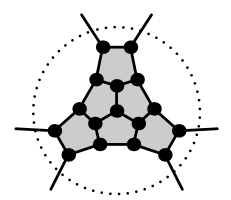

$(3,3)$

Figure 8: The caps of $\left(p_{1}, p_{2}\right)$-nanotubes, where $p_{1}+p_{2}=6$. 
Now, let us state the main theorem of this article.

Theorem 4 A fullerene graph has a non-degenerated cyclic 7-edge-cut if and only if it is a nanotube of type $\left(p_{1}, p_{2}\right)$, where

(a) $p_{1}+p_{2}=7$; or

(b) $p_{1}+p_{2}=6$, and the nanotube is not the smallest nanotube of types $(3,3)$, and $(4,2)$, or it is not of type $(6,0)$ with one layer of hexagons and with both caps isomorphic to the first configuration on Fig. 8; or

(c) $p_{1}+p_{2}=5$, with at least 2 layers of hexagons;

unless it is isomorphic to one of the two graphs depicted in Fig. 9.
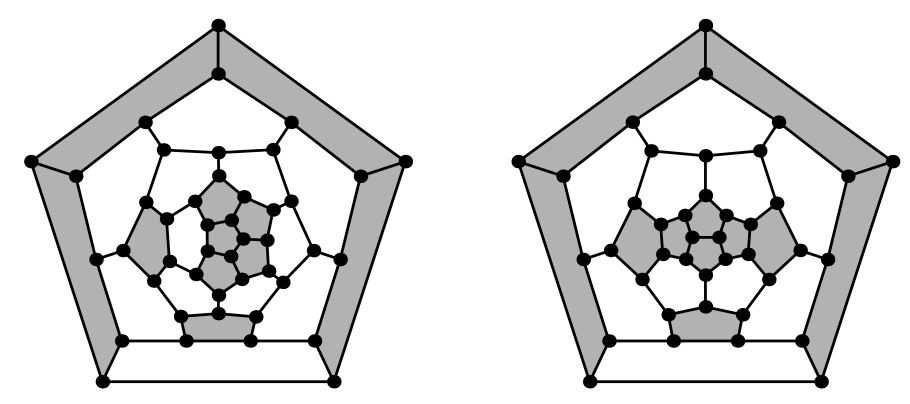

Figure 9: The only two non-nanotubical fullerenes with a 7-edge-cut.

Proof. Let $G$ be a fullerene graph and $C$ a non-degenerated cyclic 7-edge-cut in $G$. Let $H$ be one of the components of graph $G-C$. If $C$ is nanotubical, it is obvious that $G$ is a nanotube. Let us firstly consider such cuts.

We prove that all nanotubes, which contain cyclic 7-edge-cuts, are of type $\left(p_{1}, p_{2}\right)$, where $p_{1}+p_{2}=k, k \in\{5,6,7\}$. Consider cases regarding $k$. Let $k=5$ and let the cylindrical part of the nanotube have only one layer of hexagons. Then, the only edges not adjacent to pentagons are the edges between hexagonal faces. There are only five such edges, thus a cyclic 7-edge-cut could not be obtained. On the other hand, having two or more layers, the edges between layers could be used to obtain the cut of greater length.

Now, let $k=6$ and consider nanotubes of types $(5,1),(4,2),(3,3)$, and $(6,0)$ separately. The nanotubes of type $(5,1)$ have uniquely defined caps, which contain a hexagon, so all such nanotubes have a configuration on Fig. 10, where exists a nondegenerated cyclic 7-edge-cut.

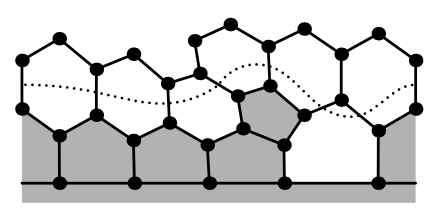

Figure 10: The cap of a nanotube of type $(5,1)$ with a non-degenerate cyclic 7 -edge-cut. 
On the other hand, the unique caps of nanotubes of types $(4,2)$ and $(3,3)$ do not contain any hexagonal faces. So there exist nanotubes of such types that do not have non-degenerate cyclic 7-edge-cut. In fact for each type only the smallest nanotube is such, while all others have it. On Fig. 11 the smallest two nanotubes of each type are presented.
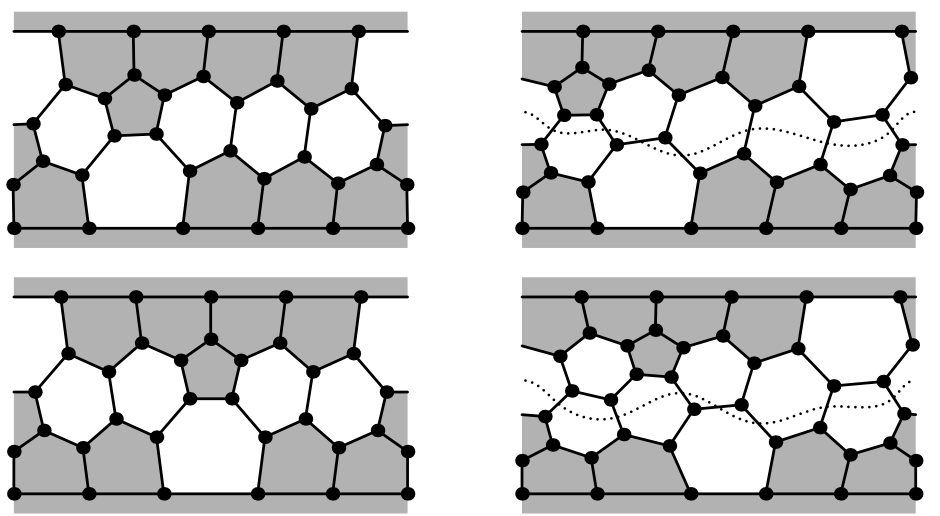

Figure 11: The two smallest nanotubes of types $(4,2)$ (on the top), and $(3,3)$ (at the bottom).

It remains to consider the nanotubes of type $(6,0)$. There are five possible caps for this type, see Fig. 8. Only the first cap does not contain a hexagonal face, so the nanotubes with both such caps need at least two layers of hexagons to obtain a nondegenerate cyclic 7-edge-cut. In all other configurations there are at least two edges in the cap that are not adjacent to a pentagonal face (the edges of cap's hexagon), and can be elements of the cut. In case, when $k=7$, simply the edges in cyclindrical part are used to obtain a cyclic 7-edge-cut.

Now, let $C$ be non-nanotubical non-degenerated 7-edge cut. Consider the cut-vector of $C$. If it contains any 3 , the complement must contain -1 , since the cut is nondegenerated. If there is a -1 , it corresponds to a vertex of degree 1 in one of the components; anytime the cut vector looks like $[\ldots, a,-1, b, \ldots]$, if we remove the vertex from the component, we get a non-degenerate cyclic 6-edge cut, with the cut vector $[\ldots,(a-1),(b-1), \ldots]$, see Fig. 12 for illustration. By Theorem 3, it is contained in a nanotube, moreover, if we insert the removed vertex back, we get a non-degenerated 7-edge-cut in the nanotube.
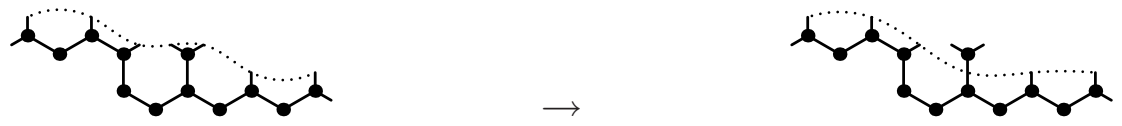

Figure 12: If the cut-vector of a $k$-cut contains -1 , we can change it into a $(k-1)$-cut.

Therefore, we deal only with $0 \mathrm{~s}, 1 \mathrm{~s}$ and $2 \mathrm{~s}$. Then, due to the definition, we have at least two consecutive 0's or 2's. So, the type of the cut-vector is one of the following three: $[2,2,2,0,0,0],[2,2,0,2,0,0]$ or $[2,2,0,0]$. Table 2 lists all possible cut-vectors (up to symmetry) which could arise from these types.

Now, we will consider each of the cut-vectors separetly and prove that any cut with such a cut-vector is either a part of a nanotube, part of the graphs depicted in Fig. 9, 


\begin{tabular}{c|c|c}
{$[2,2,2,0,0,0]$} & {$[2,2,0,2,0,0]$} & {$[2,2,0,0]$} \\
\hline$[2,2,2,1,0,0,0]$ & {$[2,1,2,0,2,0,0]$} & {$[2,2,1,1,1,0,0],[2,2,1,1,0,0,1]$} \\
{$[2,1,2,2,0,0,0]$} & {$[2,2,1,0,2,0,0]$} & {$[2,1,2,1,1,0,0],[2,1,2,1,0,0,1]$} \\
& {$[2,2,0,1,2,0,0]$} & {$[2,1,1,2,1,0,0],[2,1,2,1,0,1,0]$} \\
& {$[2,2,0,2,0,0,1]$} & {$[2,1,1,1,2,0,0],[2,1,1,2,0,1,0]$}
\end{tabular}

Table 2: All possible cut-vectors that arise from non-nanotubical cut types.

or a part of a configuration, which is non-realizable. Notice that the cuts are depicted with the dotted lines on figures.

$[\mathbf{2}, \boldsymbol{2}, \boldsymbol{2}, \mathbf{1}, \boldsymbol{0}, \boldsymbol{0}, \boldsymbol{0}]$ : Consider the configuration of Fig. 13, left. Notice that the face $A$ cannot be pentagonal, otherwise there would be a cyclic 3-edge-cut, which is impossible [3]. Thus, it is of length 6 , and we obtain a non-degenerated 5-edge-cut with a cut-vector $[2,2,0,0,1]$. But by Theorem 1 it follows that such a configuration is non-realizable, since the only cut-vector of non-degenerated 5 -edge-cut is $[1,1,1,1,1]$.

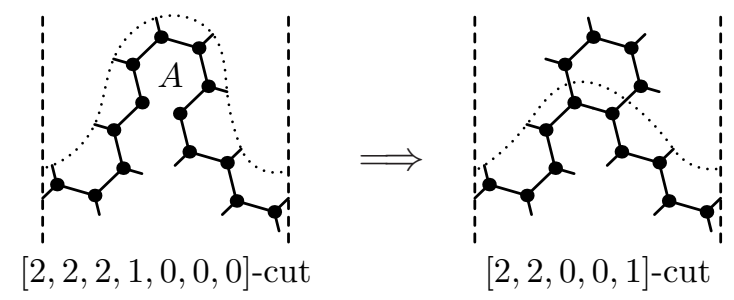

Figure 13: The component associated with the cut-vector $[2,2,2,1,0,0,0]$.

$[\mathbf{2}, \mathbf{1}, \boldsymbol{2}, \boldsymbol{2}, \boldsymbol{0}, \boldsymbol{0}, \boldsymbol{0}]$ : Consider the configuration of Fig. 14, left. Similarly as in the case above, we may see that face $A$ is of length 6 . We obtain a non-degenerated 5-edgecut with a cut-vector $[2,1,0,1,1]$. Theorem 1 implies that such a configuration is non-realizable.

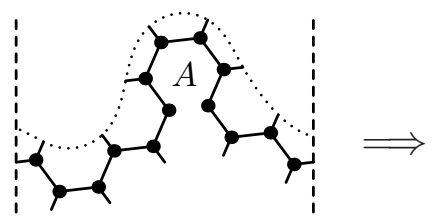

$[2,1,2,2,0,0,0]$-cut

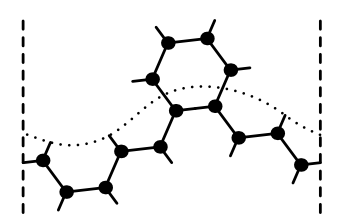

$[2,1,0,1,1]$-cut

Figure 14: The component associated with the cut-vector $[2,1,2,2,0,0,0]$.

$[\mathbf{2}, \mathbf{1}, \boldsymbol{2}, \boldsymbol{0}, \boldsymbol{2}, \boldsymbol{0}, \boldsymbol{0}]$ : Consider the size of the face $A$ from Fig. 15. If $A$ is pentagonal, we obtain a degenerated 6 -edge-cut with the cut-vector $[2,0,1,0,1,1]$. Such a configuration is non-realizable by Theorem 2 , since the cut-vectors of degenerated 6-edge-cuts with a component containing five pentagons are $[2,0,1,1,1,0]$ and $[0,1,1,1,1,1]$. On the other hand, if $A$ is hexagonal, we obtain a nanotubical cut with the cut vector $[1,1,1,2,0,1]$. 


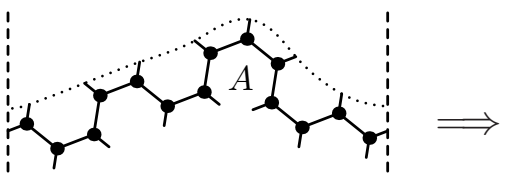

$[2,1,2,0,2,0,0]$-cut

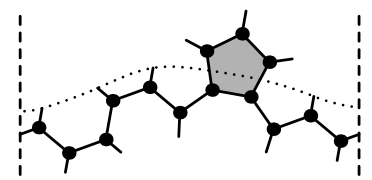

$[2,0,1,0,1,1]$-cut

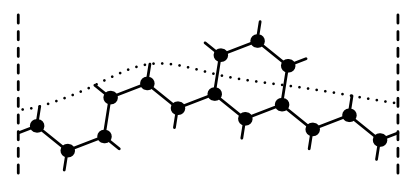

$(5,1)$-nanotube

Figure 15: The component associated with the cut-vector $[2,1,2,0,2,0,0]$.

$[2,2,1,0,2,0,0]:$ In this case the size of the face $A$ from Fig. 16, left, is considered again. If it is of size five, the configuration is non-realizable, since a degenerated 6-edgecut with the cut-vector $[2,1,0,1,0,1]$ is obtained. There is no such a degenerated cut according to Theorem 2 . If $A$ is hexagonal, we obtain a cut with the cut-vector $[2,1,0,1,1,1]$, which is nanotubical.

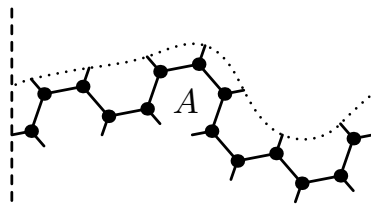

$[2,2,1,0,2,0,0]$-cut

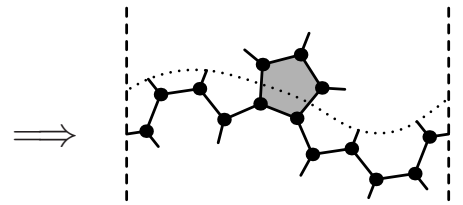

$[2,1,0,1,0,1]$-cut

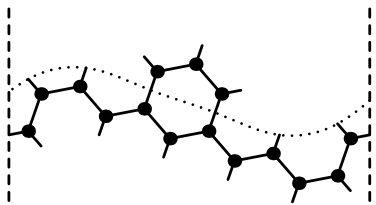

$(4,2)$-nanotube

Figure 16: The component associated with the cut-vector $[2,2,1,0,2,0,0]$.

[2,2,0,1, 2, 0,0]: Similarly as in the two cases above the size of the face $A$ from Fig. 17, left, is taken in consideration. For $A$ pentagonal we once again obtain a nonrealizable configuration, due to a cut with the cut-vector $[2,0,1,1,0,1]$. For $A$ hexagonal the nanotubical cut with the cut-vector $[2,0,1,1,1,1]$ is obtained.

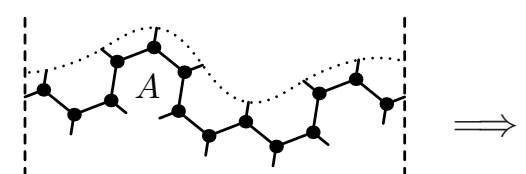

$[2,2,0,1,2,0,0]$-cut

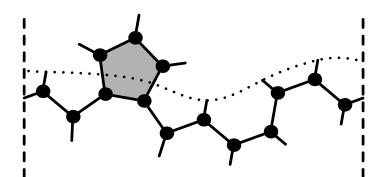

$[2,0,1,1,0,1]$-cut

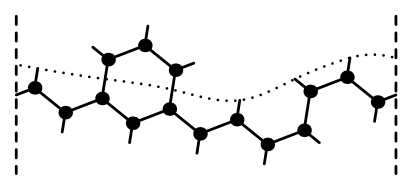

$(5,1)$-nanotube

Figure 17: The component associated with the cut-vector $[2,2,0,1,2,0,0]$.

[2, 2, 0,2,0,0,1]: Analogously, if the face $A$ from Fig. 18, left, is pentagonal, we once again obtain a non-realizable cut-vector $[2,2,0,1,0,0]$. If $A$ is hexagonal, a nondegenerate cyclic 6 -edge-cut with the cut-vector $[2,2,0,1,1,0]$ is obtained. By Theorem 3 it must be nanotubical.

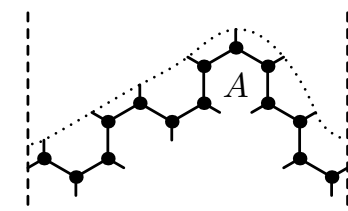

$[2,2,0,2,0,0,1]$-cut

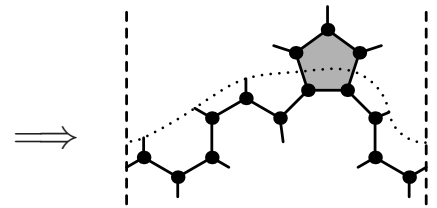

$[2,2,0,1,0,0]$-cut

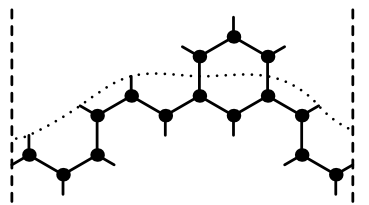

$(5,0)$-nanotube

Figure 18: The component associated with the cut-vector $[2,2,0,2,0,0,1]$. 
$[\mathbf{2}, \mathbf{2}, \mathbf{1}, \mathbf{1}, \mathbf{1}, \boldsymbol{0}, \boldsymbol{0}]:$ If the face $A$ from Fig. 19, left, is pentagonal, we obtain a degenerated cyclic 6 -edge-cut with a cut-vector $[2,1,1,0,0,1]$ which is non-realizable. If $A$ is hexagonal, we obtain a nanotubical cut-vector $[1,2,1,1,0,1]$.

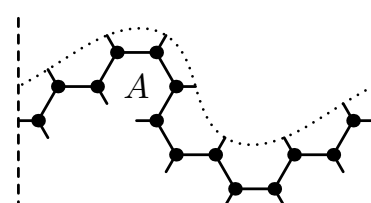

$[2,2,1,1,1,0,0]$-cut

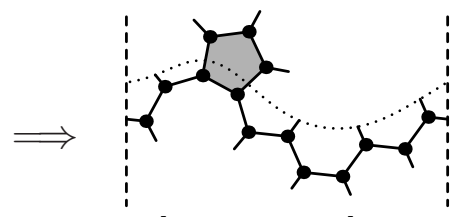

$[2,1,1,0,0,1]$-cut

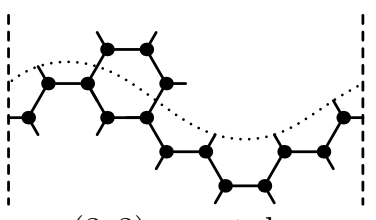

$(3,3)$-nanotube

Figure 19: The component associated with the cut-vector $[2,2,1,1,1,0,0]$.

[2, 2, 1, 1, 0,0,1]: Consider the face $A$ from Fig. 20, left. If $A$ is pentagonal, we obtain a degenerated 6 -edge-cut with the cut-vector $[2,2,1,0,0,0]$, which is non-realizable. If $A$ is hexagonal, we obtain a non-degenerated 6-edge-cut, which is by Theorem 3 nanotubic. (However, it can be easily checked that it is non-realizable, too, since it leads to a nanotube of type $(4,1)$, which does not exist [4].)

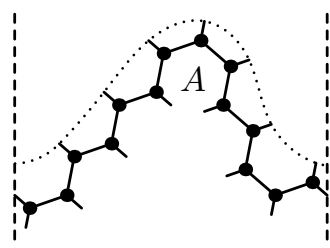

$[2,2,1,1,0,0,1]$-cut

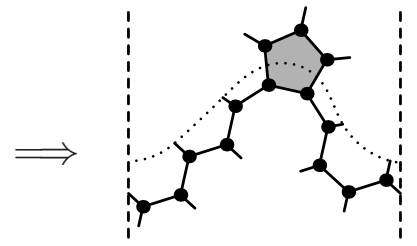

$[2,2,1,0,0,0]$-cut

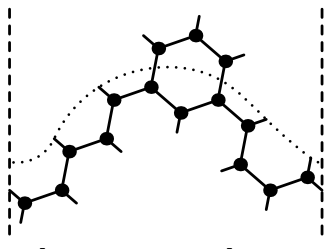

$[2,2,1,0,1,0]$-cut

Figure 20: The component associated with the cut-vector $[2,2,1,1,0,0,1]$.

[2, 1, 2, 1, 1, 0,0]: Consider the face $A$ from Fig. 21, left. If it is pentagonal, we obtain a cut with the cut-vector $[2,1,0,0,1,1]$, which is non-realizable by Theorem 2 . If the face $A$ is hexagonal, we obtain a cut with a nanotubical cut-vector $[2,1,0,1,1,1]$.

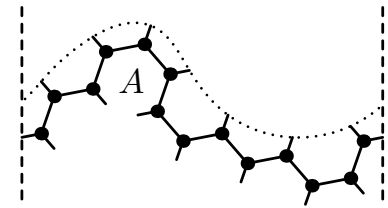

$[2,1,2,1,1,0,0]$-cut

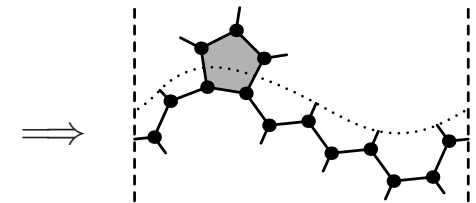

$[2,1,0,0,1,1]$-cut

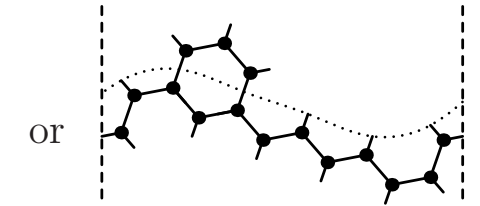

$(4,2)$-nanotube

Figure 21: The components associated with the cut-vector $[2,1,2,1,1,0,0]$.

$[\mathbf{2}, \mathbf{1}, \mathbf{2}, \mathbf{1}, \boldsymbol{0}, \boldsymbol{0}, \mathbf{1}]:$ Consider the face $A$ from Fig. 22, left. If $A$ is pentagonal, we obtain a degenerated 6 -edge-cut with the cut-vector $[2,1,2,0,0,0]$, which is non-realizable. If $A$ is hexagonal, we obtain a non-degenerated 6 -edge-cut with the cut vector $[2,1,2,0,1,0]$, which can only appear in a nanotube.

$[\mathbf{2}, \mathbf{1}, \mathbf{1}, \boldsymbol{2}, \mathbf{1}, \boldsymbol{0}, \boldsymbol{0}]:$ Consider the face $A$ from Fig. 23, left. If it is pentagonal, we obtain a cut with the cut-vector $[2,0,0,1,1,1]$, which is non-realizable by Theorem 2 . If the face $A$ is hexagonal, we obtain a cut with a nanotubical cut-vector $[2,0,1,1,1,1]$. 


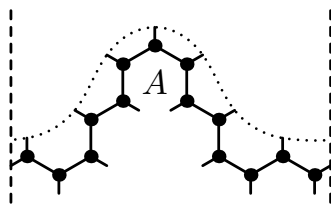

$[2,1,2,1,0,0,1]$-cut

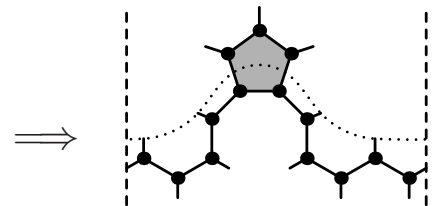

$[2,1,2,0,0,0]$-cut

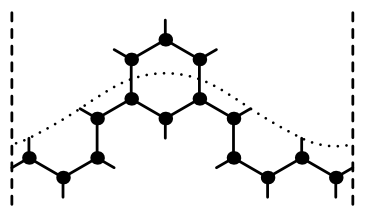

(5, 0)-nanotube

Figure 22: The component associated with the cut-vector $[2,1,2,1,0,0,1]$.

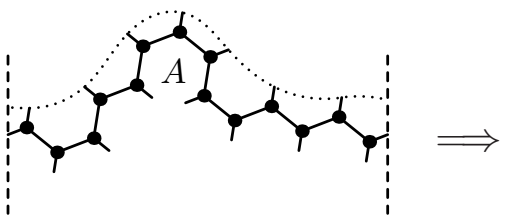

$[2,1,1,2,1,0,0]$-cut

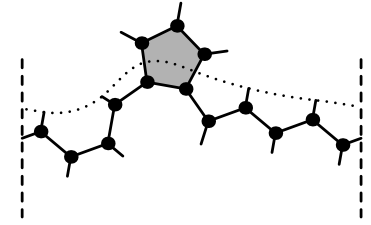

$[2,0,0,1,1,1]$-cut

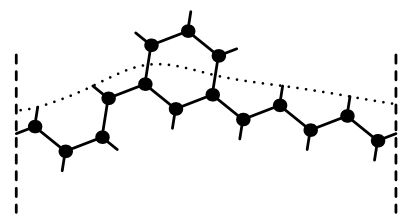

(5, 1)-nanotube

Figure 23: The components associated with the cut-vector $[2,1,1,2,1,0,0]$.

[2, 1,2, 1, 0, 1,0]: Consider the face $A$ from Fig. 24, left. If $A$ is pentagonal, we obtain a degenerated 7-edge-cut with a component of five pentagons and some hexagons, with the cut-vector $[2,1,2,0,1,0,0]$, which is non-realizable, since no degenerated 7-edge-cut in Table 1 has such cut-vector. If $A$ is hexagonal, we obtain a nondegenerated 7 -edge-cut with the cut vector $[2,1,2,0,2,0,0]$, which has already been considered and leads to nanotubic cuts only.

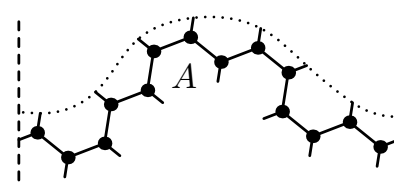

$[2,1,2,1,0,1,0]$-cut

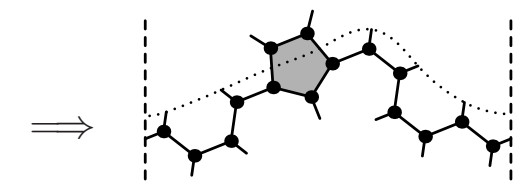

$[2,1,2,0,1,0,0]$-cut

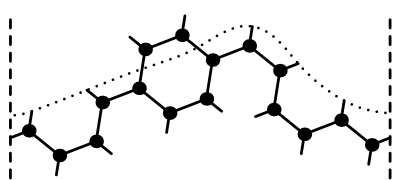

$[2,1,2,0,2,0,0]$-cut

Figure 24: The component associated with the cut-vector $[2,1,2,1,0,1,0]$.

$[2, \mathbf{1}, \mathbf{1}, \mathbf{1}, \mathbf{2}, \mathbf{0}, \mathbf{0}]:$ In this case we consider two sub cases again, starting with the case with $A$ being hexagonal. In that case we obtain a 6 -edge-cut with the cut-vector $[1,1,1,1,1,1]$ (see Fig. 25), which is nanotubical.

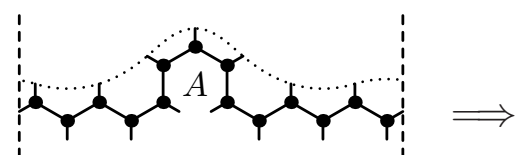

$[2,1,1,1,2,0,0]$-cut

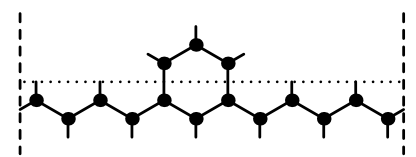

$(6,0)$-nanotube or

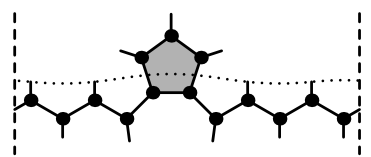

$[0,1,1,1,1,1]$-cut

Figure 25: The component associated with the cut-vector $[2,1,1,1,2,0,0]$.

In the latter case $A$ is pentagonal. We obtain a degenerated 6-edge-cut with the cut-vector $[0,1,1,1,1,1]$. By Theorem 2 , we know that there exists precisely one configuration with such a cut. It is composed by five pentagons and one hexagon, which is by the component with 0 value in the cut. We obtain the left configuration on Fig. 26. Obviously, it is realizable and does not have to be nanotubical, so we have to consider the other part of the graph, the complement of the original cutvector - $[0,1,1,1,0,2,2]$. 
Consider the faces $A, B, C$ and $D$ on Fig 26, left. We distinguish cases regarding their sizes. Notice that in all cases we obtain a cut with the cut-vector, which has two consecutive components with value 1 . When all four faces are hexagonal, we obtain a nanotubical 6 -edge-cut with the cut-vector $[1,1,1,1,1,1]$. When at least one of them is pentagonal, a degenerated cut is obtained. By the Theorem 2 and the fact that there are two consecutive 1's in the cut-vector of the cut passing the faces $A, B, C, D$, and the two topmost hexagons drawn in Fig 26, it follows that either one or two faces are pentagonal. When only one of the faces is pentagonal, we consider two subcases, due to the symmetry, either $A$ is pentagonal or $B$ is pentagonal.

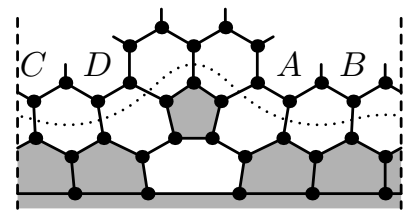

$[0,1,1,1,0,2,2]$-cut

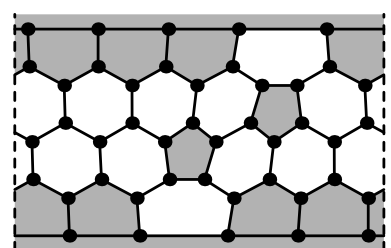

the first exception

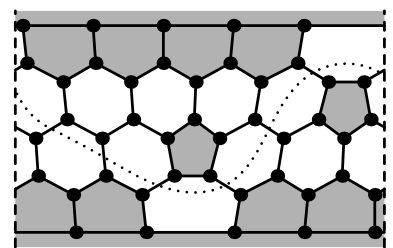

$(4,3)$-nanotube

Figure 26: The components associated with the cut-vector $[0,1,1,1,0,2,2]$ : the general situation and the cases when only $A$ or $B$ is pentagonal.

If the face $A$ is pentagonal, we obtain a 6 -cut with cut-vector $[0,1,1,1,1,1]$, which is realizable uniquely. We get the middle graph drawn in Fig 26, which is isomorphic to the left graph of Fig. 9. There is no nanotubical cut in it, so this fullerene is not a nanotube.

If the face $B$ is pentagonal, we again obtain a 6 -cut with cut-vector $[0,1,1,1,1,1]$, which is realizable uniquely. We get the right graph draw in Fig 26. It is a nanotube of type $(4,3)$.

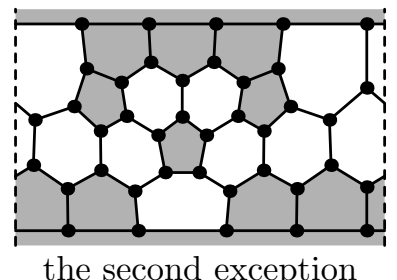

Figure 27: The graph obtained from the cut-vector $[0,1,1,1,0,2,2]$ in the case two of the faces $A, B, C, D$ are pentagonal.

In the latter case precisely two of the faces $A, B, C$ and $D$ are pentagonal. We obtain a degenerated cut with four 5 -faces in the interior. The only such configuration has the cut-vector $[1,1,0,1,1,0]$. Notice that between the 0 components are two 1's. That infers the pentagonal faces are $A$ and $D$, since there must be exactly two hexagons between the pentagons. The configuration is again realizable. We obtain the graph depicted in Fig. 27, which is isomorphic to the right graph of Fig. 9. It is not a nanotube, since there is no nanotubical cut in it. 
$[\mathbf{2}, \mathbf{1}, \mathbf{1}, \mathbf{2}, \boldsymbol{0}, \mathbf{1}, \boldsymbol{0}]:$ Consider the faces $A$ and $B$ on Fig. 28, left. If both of them are hexagonal, we obtain a cut with the cut-vector $[1,1,1,1,1,1]$, therefore it is nanotubical. If at least one of them is pentagonal, we obtain a degenerated cut with the cut-vector having three consecutive 1's. The only degenerated cut with the cut-vector having three consecutive 1's has five pentagons in the interior, so exactly one of the faces $A$ and $B$ is pentagonal. In that case, we can always find a cut with the cut-vector $[2,1,1,1,2,0,0]$, see Fig. 28 . Therefore, we deal only with configurations already mentioned above.

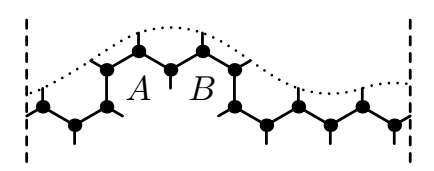

$[2,1,1,2,0,1,0]$-cut

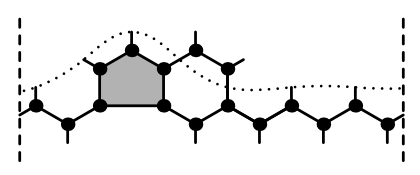

$[2,1,1,1,2,0,0]$-cut

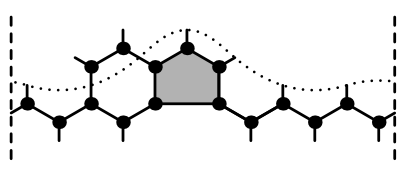

$[2,1,1,1,2,0,0]$-cut

Figure 28: The components associated with the cut-vector $[2,1,1,2,0,1,0]$.

This proves the theorem.

Acknowledgement. Supported in part by bilateral projects BI-SK/05-07-001 and SK-SI-0007-08 between Slovenia and Slovakia, by Slovak Research and Development Agency under the contract No. APVV-0007-07, by the Slovak VEGA Grant 1/3004/06, and by the Slovenian ARRS Research Grant P1-0297.

\section{References}

[1] T. Došlić, On lower bounds of number of perfect matchings in fullerene graphs, J. Math. Chem. 24 (1998) 359-364.

[2] T. Došlić, On some structural properties of fullerene graphs, J. Math. Chem. 31 (2002) 187-195.

[3] T. Došlić, Cyclical edge-connectivity of fullerene graphs and $(k, 6)$-cages, J. Math. Chem. 33 (2003) 103-112.

[4] F. Kardoš and R. Škrekovski, Cyclic edge-cuts in fullerene graphs, J. Math. Chem. 22 (2008) 121-132.

[5] D. J. Klein and X. Liu, Theorems for carbon cages, J. Math. Chem. 33 (1992) 199-205.

[6] K. Kutnar and D. Marušič, On cyclic edge-connectivity of fullerenes, Discrete App. Math. 156(10) (2008) 1661-1669.

[7] J. Qian and F. Zhang, On the number of Kekule structures in capped zigzag nanotubes, J. Math. Chem. 38 (2005) 233-246.

[8] F. Zhang and L. Wang, k-resonance of open-ended carbon nanotubes, J. Math. Chem. 35 (2004) 87-103. 